\title{
Biomedical implications of viral mutation and evolution
}

\author{
Rafael Sanjuán ${ }^{1}$, Ester Lázaro $^{2}$, Marco Vignuzzi ${ }^{3}$ \\ ${ }^{1}$ Instituto Cavanilles de Biodiversidad y Biología Evolutiva, Universitat de \\ València, Valencia, Spain. E-mail: rafael.sanjuan@uv.es \\ ${ }^{2}$ Centro de Astrobiología, INTA-CSIC, Madrid, Spain. E-mail: lazarolm@inta.es \\ ${ }^{3}$ Viral Populations and Pathogenesis Laboratory, Institut Pasteur, Paris, France. E- \\ mail: marco.vignuzzi@pasteur.fr
}




\begin{abstract}
Mutation rates vary hugely across viruses and strongly determine their evolution. In addition, viral mutation and evolution are relevant to several fields of biomedicine, including the study of pathogenesis, vaccine design or antiviral research. In this review, we outline how viral mutation rates are estimated and how they vary across viral groups, paying special attention to general trends. We review recent advances positing a direct association between viral mutation rates and virulence, or the use of high fidelity variants as attenuated vaccines. The implications of mutation rates for the design of rational antiviral therapies and for efficient epidemiological surveillance are also discussed.
\end{abstract}

Keywords: mutation rate, replication fidelity, evolutionary biology, viral escape, viral fitness, lethal mutagenesis, vaccine. 


\section{Main text}

Viruses are amongst the fastest evolving entities, with several nucleotide substitutions occurring in the global population each year in most RNA and some DNA viruses $[1,2]$. This implies that the genetic diversity produced in a few years of viral evolution is often equivalent to millions years of evolution in their eukaryotic hosts. The mutation rate of an organism is defined as the per-generation per-nucleotide probability of erroneous replication. Since viral generations are poorly defined, the mutation rate is expressed per replication cycle or per cell infection cycle [3]. Viruses show highly variable mutation rates and, therefore, widely different evolutionary properties [1]. Although there are other important factors determining evolution such as generation times, population sizes, selection strength, or recombination rates, mutation is of particular interest because it is the ultimate source of genetic variation.

Viral mutation has important biomedical implications. For instance, the high mutation rate of HIV-1 implies that any single substitution conferring drug resistance takes place within a single day and makes multidrug treatments necessary [4], an argument that applies similarly to other RNA viruses producing chronic infections such as hepatitis $\mathrm{C}$ virus (HCV). The viral mutation rate also plays a role in the assessment of vaccination strategies [5] and influences the stability of live attenuated vaccines [6]. Additionally, mutation rates can determine viral fitness and virulence $[7,8]$ and thus the ability of the host immune system to clear the infection. At the epidemiological level, the viral mutation rate can determine the risk of emergence of new diseases $[2,9]$

This review focuses on the evolutionary and biomedical implications of viral mutation. Firstly, we briefly outline some mechanistic aspects of viral mutation and review a few methods for measuring mutation rates. Secondly, we summarize the general trends emerging from current mutation rate data. Finally, we discuss the 
relevance of viral mutation to pathogenesis, vaccination strategies, and antiviral research.

\section{Measuring viral mutation rates}

There are three basic approaches to determining viral mutation rates: cell-free replication fidelity assays (in vitro), cell culture systems (ex vivo), and analysis of samples taken directly from hosts (in vivo). Abundant in vitro information is available for HIV-1 and other RNA viruses from studies of the incorporation kinetics of correct and incorrect bases and from replication assays using reporter sequences [10-12]. These have provided invaluable insights into the mechanistic aspects of replication fidelity. A salient result is that all viral RNA-dependent RNA polymerases (RdRp) except those of coronaviruses and all reverse transcriptases (RT) studied to date lack 3 'exonuclease proofreading activity and incorporate mismatches into the nascent chain relatively efficiently [10,13-15] However, this does not imply that viral RdRps and RTs lack any mechanism of fidelity. Their base-selection specificity is similar to that of cellular DNA polymerases and key amino acids involved in fidelity have been identified $[10,16]$. The sequence context of the template is also important. For instance, templateprimer misalignment at homopolymeric runs can cause frameshift mutations or nucleotide substitutions [17].

However, the mutation rates inferred in vitro tend to differ substantially from those obtained in cell cultures and qualitative differences exist as well. One possible explanation is that in vitro assays use saturating amounts of essential compounds such as nucleotides or divalent cations, which can affect replication fidelity and alter the mutational spectrum. For instance, in HIV-1, G-to-A is the most frequent type of mutation in vivo, but not in vitro [10]. Another reason for this inconsistency is that mutations depend on factors other than the viral polymerase. In retroviruses, dUTPases 
reduce mutation levels by preventing the incorporation of uracil into DNA [18], and the Vpr protein is known to recruit the host-encoded uracil DNA glycosylase UNG2, a protein involved in DNA repair, into the virion [19]. Furthermore, mutations are also a consequence of spontaneous nucleic acid damage and host-mediated editing. Apolipoprotein B mRNA-editing catalytic polypeptide-like enzymes (APOBEC3) are cytidine deaminases that produce G-to-A hypermutation in retroviruses [20-22], and the double-stranded RNA-dependent adenosine deaminase (dsRAD) may produce A-to-G hypermutation in several RNA viruses, including rhabdoviruses [23], paramyxoviruses [24], and retroviruses [25-27].

Mutation rates have been determined in cell cultures for over twenty viruses using a variety of methods [3]. For instance, a genetically homogeneous inoculum (e.g. a plaque or an infectious clone) can be propagated for one or a few passages and the genetic variation appeared during this time interval quantified by PCR, molecular or biological cloning and sequencing of a large number of clones encompassing one or several genome regions. By dividing by the number of infection cycles elapsed, this variation can be converted to a mutation rate. However, deleterious mutations will be underrepresented relative to neutral mutations. This selection bias will increase with time and has to be corrected appropriately [3]. For some viruses, particularly retroviruses, culturing systems have been developed to allow the virus to replicate in the absence of selection, thus avoiding this problem. Mutations introduced by the polymerases used for PCR amplification are yet another source of inaccuracy in molecular clone sequencing [28]. To minimize this problem, the cloning step can be carried out before the PCR by picking isolated plaques (i.e. isolated plaques) or limiting-dilution PCR. 
An alternative approach to mutation rate estimation is the classical Luria-Delbrück fluctuation test $[3,29,30]$. To carry out this test, one has to seed a large number of parallel cultures with a small initial number of individuals each, let them replicate in a permissive medium until a given titer is reached, and plate in the presence of a selective agent such as a monoclonal antibody, an antiviral, or a non-permissive cell type, in which only certain mutants can form plaques. The distribution of the number of resistant plaques per assayed culture can then be used for estimating the mutation rate (Box 1, Fig .1). This test has the advantage of avoiding PCR errors and selection bias, but necessarily focuses on a few genome sites (i.e. those encoding the resistant phenotype).

There are very few in vivo mutation rate estimates for viruses. In two plant virus studies, a transgenic host was engineered to redundantly express one viral gene, such that the viral copy was released from selection by trans-complementation and could accumulate mutations neutrally $[31,32]$. However, this method needs an estimation of the number of viral generations elapsed in the host, which makes it essentially not applicable to human and most animal viruses. A more widely applicable strategy is to focus on mutations that suppress viral infectivity (lethal mutations). Since such mutants leave no progeny, their population frequency remains equal to the mutation rate at any time. By determining the within-host frequency of putative lethal mutations in large collections of molecular clones, it was possible to determine the in vivo mutation rate of a viroid [33] and to demonstrate that the standard ribavirin/interferon treatment against $\mathrm{HCV}$ is mutagenic for the virus [34]. However, this method requires considerable sequencing effort and careful controls to distinguish naturally occurring mutations from sequencing errors. 
In RNA viruses, mutation rates estimated in cell cultures or in vivo range from $10^{-6}$ to $10^{-4}$ substitutions per nucleotide per cell infection $(\mathrm{s} / \mathrm{n} / \mathrm{c})$, whereas the range for DNA viruses is from $10^{-8}$ to $10^{-6} \mathrm{~s} / \mathrm{n} / \mathrm{c}$ [3]. Therefore, there is little or no overlap between these two large groups (Fig. 2). The biochemical basis for this dichotomy is wellestablished because the vast majority of RNA virus polymerases lack 3 'exonuclease proofreading activity. The immediate consequence is that, generally speaking, RNA viruses evolve faster than DNA viruses [1,2]. However, there are at least two other important factors to be considered when making generalizations about viral mutation rates: genome size and whether the genome is single- or double-stranded.

Drake's rule establishes that the genomic mutation rate of DNA-based microorganisms, including viruses, is roughly constant and approximately equal to 0.003 [35]. This implies that there is an inverse relationship between the per-nucleotide mutation rate and genome size spanning several orders of magnitude. Single-stranded (ss) DNA viruses and some double-stranded (ds) DNA viruses have genome sizes below $10 \mathrm{~kb}$, implying that their per-nucleotide mutation rates are close to $10^{-6} \mathrm{~s} / \mathrm{n} / \mathrm{c}$ and thus not extremely different from those of some RNA viruses. Consistently, ssDNA viruses have been shown to evolve relatively fast [1,2]. For instance, the evolutionary rates of canine parvovirus, panleukopenia virus [36] and human B19 erythrovirus [37] are on the order of $10^{-4}$ substitutions per nucleotide site per year $(\mathrm{s} / \mathrm{s} / \mathrm{y})$, which is within the typical range of $10^{-2}$ to $10^{-5} \mathrm{~s} / \mathrm{s} / \mathrm{y}$ accepted for RNA viruses [38]. In RNA viruses, in contrast, genomic mutation rates are highly variable and evidence supporting a negative correlation between per-nucleotide mutation rates and genome sizes is weak [3]. Interestingly, however, the chrysanthemum chlorotic mottle viroid has a minimal genome of $0.4 \mathrm{~kb}$ and the highest per-nucleotide mutation rate described [33]. Furthermore, the genome of bacteriophage $Q \beta$ is one of the smallest 
among RNA viruses and its estimated mutation rate is one the highest [3]. Finally, coronaviruses, which are the RNA viruses with the largest genomes, encode for a 3 'exonuclease which is probably used for proofreading [14, 15].

Positive-stranded, negative-stranded, and double-stranded viruses differ profoundly in their replication and gene expression strategies. For instance, in ss(+)RNA viruses, the genomic RNA is used directly as mRNA, whereas in ss(-)RNA viruses the genome needs to be transcribed first. The genomes of ss $(+)$ RNA viruses interact less strongly with nucleoproteins, tend to form more RNA secondary structures, and recombine more frequently by replication template switching [39]. Given these differences, it seems reasonable that we might also see differences in mutation rates among major viral groups. Indeed, available mutation rate estimates indicate that ssDNA viruses mutate faster than dsDNA viruses (Fig. 2). Although ssDNA viruses use host-encoded DNA polymerases for replication, their relatively high mutation rates can still be explained by the lower stability of single-stranded nucleic acids or the absence of post-replicative repair. For instance, GATC motifs are required for methyl-directed mismatch repair in Echerichia coli [40], but the genome of its phage $\phi X 174$ has few or no such motifs. In fact, the mutation rate of $\phi X 174$ can be significantly reduced by adding GATCs to the viral genome [41].

In contrast, differences between ssRNA and dsRNA viruses have not been demonstrated. Mutation rates have been determined for seven ss $(+)$ RNA viruses and four ss(-)RNA viruses [3], but the only estimate for a dsRNA virus corresponds to bacteriophage $\phi 6$ [42]. Interestingly, while the median mutation rate is $7 \times 10^{-5} \mathrm{~s} / \mathrm{n} / \mathrm{c}$ for $\mathrm{ss}(+) \mathrm{RNA}$ viruses and $2 \times 10^{-5} \mathrm{~s} / \mathrm{n} / \mathrm{c}$ for ss(-)RNA viruses, the estimate for bacteriophage $\phi 6$ is only $2 \times 10^{-6} \mathrm{~s} / \mathrm{n} / \mathrm{c}$, at the lower limit of the accepted $10^{-6}-10^{-4}$ 
range. This suggests that there may be differences between ssRNA and dsRNA similar to those established for DNA viruses, but more data are required to test this hypothesis.

\section{Viral mutation and virulence}

The link between mutation rates and virulence in the microbial world has been best characterized with bacteria, for which mutator and antimutator strains have been described over three decades. Naturally occurring and genetically engineered mutator strains of numerous bacterial species have permitted in vitro and in vivo studies that reveal a positive link between mutation rate, adaptation and virulence [43-46]. In bacteria, the temporary, positive selection of hypermutators, with mutation rates up to 10000-times higher than normal [47], correlates with the development of antibiotic resistance and adaptive radiation leading to colonization and persistence in hosts. Given the large variability in the mutation rates of viruses the question of a link between mutability and virulence seems all the more pertinent. The recent isolation of variants of a number of RNA viruses presenting higher and lower fidelity RdRps has opened to the way to experimentally examine these concepts both in vitro and in vivo $[16,48]$.

Currently, the clearest connection between mutation rate and virulence has been obtained in a body of work involving the G64S high fidelity variant of poliovirus [6$8,16]$. With only a single amino acid difference from wild type virus, this variant replicated with increased fidelity and generated approximately 3-6-fold fewer mutations; while retaining wild type-like replication kinetics and all of the known determinants for pathogenesis. The resulting decrease in genetic diversity generated by this variant was shown to correlate with a loss in adaptability, in the ability to generate resistance mutations to antiviral compounds and escape mutants to neutralizing antibody. Most surprisingly, despite making more perfect copies of the pathogenic sequence of wildtype poliovirus, this virus population was found to be moderately [7] to 
highly [8] attenuated in the murine infection model. The researchers proposed that, in order to successfully infect a host, an RNA virus must maintain a high mutation rate to adapt to changing conditions within the host environment (e.g. cell type, tissue tropism) and that even a small decrease in this mutability could have dramatic fitness costs in vivo. A recent confirmation of this observation was obtained with a higher fidelity variant of chikungunya virus that also presented wildtype-like replication kinetics but reduced mutation frequencies. In this case, infection and dissemination in the natural mosquito host was compromised and viremia in the mammalian model was negatively impacted [49]. These works reveal that moderate changes in mutation rates of RNA viruses can result in significant fitness costs. Interestingly, a low fidelity polymerase of poliovirus has been generated in vitro that is non-viable in vivo, suggesting that increasing mutation rates beyond the already high levels of natural RNA virus populations may be have equally negative effects [50] (see below).

\section{Viral mutation and vaccines}

Although innate, non-specific immunity plays an essential role in blocking viral infection and limiting replication and dissemination, it is the adaptive, specific immune responses that are required for virus clearance and long-term protection from future infections. Since adaptive immune responses recognize specific peptide sequences or protein structures, RNA viruses have limited options available to them to keep ahead of developing immunity. Some have acquired the ability to down-regulate some branches or effectors of immunity, but their small genomes and repertoire of proteins cannot host the immune evasion genes that are found in large DNA viruses or larger non-viral microbes. Thus, they must largely rely on their mutation rates to introduce amino acid changes at key antigenic sites that abrogate recognition by pre-existing immunity. This mutational drift is well documented, for example, in seasonal influenza viruses where 
the surface glycoproteins targeted by the antibody response undergo continual antigenic change [51]. The influenza vaccines therefore, must be updated annually to take into account this change, since differences approaching 5\% at antigenic sites result in little or no cross-protection. Further complicating this is the global variability of the virus, where geographic regions may not harbor the same antigenic variants and vaccine composition must be locally adapted. Other key examples where the interplay between mutation rates and immune selective pressures driving virus evolution have strong implications for vaccination include the viruses HIV and HCV, chronically infecting viruses that present both a huge global variability in the strains that circulate, as well as a considerable within-host variability of antigenic variants. In these chronic infections, the development of a universal vaccine against all strains is a very challenging task [52].

Given this key obstacle in vaccine design, a better understanding of a specific virus' mutation rate, how to best measure it and how to manipulate it would widen the options on the rational design of vaccines or other antiviral strategies. The recent work on higher fidelity variants of RNA viruses that reveal an attenuating effect suggests that increasing replication fidelity could constitute a novel component in the development of live virus vaccines. The high fidelity variants of poliovirus, for example, were completely attenuated in mice and protected against lethal challenge by homologous virus up to six months post immunization [6]. The potential safety benefits of adding such a component to live virus vaccines are two-fold: the virus, being less adaptable, is seemingly compromised in its ability to disseminate to secondary tissues and be shed into the environment by the vaccinated individual, and the likelihood of accumulating new virulent mutations or reverting other attenuating mutations to wildtype sequences is significantly reduced. Such features are particularly desired for live vaccines such as 
the poliovirus Sabin strains, for which vaccine-derived virus shed by vaccinees frequently carry reverted sequences that are pathogenic to naïve hosts [53].

\section{Viral mutation and antiviral therapies}

Antiviral drugs inhibit some essential step in the viral infection cycle such as attachment and entry, uncoating, replication, integration into the host genome in retroviruses, or virus release [54]. Many of these drugs are highly effective in the shortterm but drug-resistant viruses evolve in most cases making the treatment unsuccessful $[55,56]$. Indeed, due to their effectiveness, antiviral drugs impose a strong selective pressure to the virus. Therefore, once a mutation conferring drug resistance has appeared its population frequency increases fast. Since many resistance mutations only involve a single amino acid change, the waiting time until the mutation appears is often short, particularly in RNA viruses. Furthermore, the high mutation rates of RNA viruses make that the viral population infecting a single individual usually harbors all possible single-nucleotide mutants and many double such mutants [57]. Therefore, resistance mutants often exist before the antiviral treatment starts, albeit as minority variants. Although most resistance mutations have a fitness cost for the virus, evolution in the continuous presence of the inhibitor permits the selection of secondary mutations that compensate this cost, which facilitates the transmission of resistant virus among individuals $[58,59]$.

In light of the above facts, a successful strategy to diminish the spread of resistant mutants is the use of combination therapies where several drugs that inhibit different steps of the virus infection cycle are simultaneously administered, thus making resistance less likely. The clearest example of the advantages provided by combination therapies is represented by the highly active antiretroviral therapy (HAART) for HIV [60]. HAART regimes include one or two nucleoside reverse transcriptase inhibitors, 
one non-nucleoside reverse transcriptase inhibitor, and an additional drug that is usually a protease inhibitor. Combination therapy is also used to treat $\mathrm{HCV}$.

The high mutation rates of RNA viruses make them highly adaptable to new environments, including those produced by antiviral therapies. However, the relationship between mutation and adaptation is not straightforward. Increasing the mutation rate accelerates adaptation by reducing the waiting time before the appearance of advantageous mutations. However, a very high mutation rate can hamper adaptation because beneficial mutations will tend to occur in genetic backgrounds loaded with deleterious mutations and thus fail to spread in the viral population [61]. The reason for this interference is that deleterious mutations are universally more likely than advantageous ones. This sets the stage for an antiviral strategy called lethal mutagenesis, which consists of increasing the mutation burden of a population using specific mutagens $[57,62,63]$. Since spontaneous mutation rates are highest among RNA viruses, the latter are the best candidates for this kind of therapy.

The idea of lethal mutagenesis as a feasible goal in RNA viruses was first inspired by the quasispecies theory, which explores the population dynamics of highly error prone self-replicating molecules and predicts an error threshold beyond which genetic information cannot be sustained [64]. Early experiments demonstrated that slight increases in the replication error rate of poliovirus and vesicular stomatitis virus were associated to strong decreases in viral infectivity [65]. Lethal mutagenesis has proved effective in cell cultures and animal models against several RNA viruses [62] including enteroviruses [66,67], aphtoviruses [68], hantaviruses [69], arenaviruses [70], and lentiviruses [71,72], and is currently being tested in patients [73]. Interestingly, ribavirin, a nucleoside analogue used to treat several viral diseases, is a mutagen for 
RNA viruses in cell cultures [66] and this mutagenic effect has also been shown in vivo in patients treated with interferon and ribavirin [34].

Sequence analysis of populations at stages previous to extinction through lethal mutagenesis shows a progressive increase in both the mutation frequency and the complexity of the mutant spectrum as a function of the number of generations experienced in the presence of the mutagen $[68,74]$, even if the average or consensus sequence of the virus may remain unchanged [75] (Fig. 3). Studies carried out with lymphochoriomeningitis virus [70] and foot-and-mouth disease virus (FMDV) [76] have also shown that, at increased mutation rates, defective genomes become frequent and can parasitize functional viruses, favouring the extinction of the whole population [70]. Therefore viral extinction through mutagenesis can be a complex process [77].

Mutagenesis can also lead to the emergence of resistances. This was first demonstrated when poliovirus passaged in the presence of ribavirin evolved a single amino acid substitution that increased the overall replication fidelity of the virus [16]. Fidelity variants have also been isolated in coxsackie virus replicating in the presence of different mutagens [48]. In FMDV, in contrast, resistance to ribavirin was attained through a mutation that increased the $\mathrm{RdRp}$ ability to discriminate between the correct nucleotide and the base analog [78]. This mutant, when exposed to higher ribavirin concentrations, evolved another resistance mechanism consisting in the modulation of the types of mutations induced by the mutagen [79]. Recently, resistance to azacytidine in bacteriophage $\mathrm{Q} \beta$ has been associated with the fixation of a substitution in the readthrough protein of the virus, which is not involved in replication [80]. Finally, another mechanism that could be used by RNA viruses to avoid the negative effects of mutagenesis is increased mutational robustness [81]. To minimize the probability of resistance, mutagens could be combined with other antiviral drugs, since theory predicts 
that this increases the probability of viral clearance [63]. Recent work suggests that a sequential treatment first with the inhibitor and then with the mutagen may be more effective than the administration of both compounds in combination [82].

\section{Viral mutation, evolution, and epidemiology}

In fast evolving RNA viruses, epidemiological and evolutionary processes take place in the same timescales, and isolates taken only months apart or sometimes even days apart undergo enough genetic divergence to infer their phylogenetic relationships with confidence $[83,84]$. One practical implication is that molecular epidemiology and phylogenetic analysis can be used to address forensic issues. For instance, this made it possible to assess the role of medical staff in transmitting HIV-1 to patients in a trial case in Lybia [85] or to demonstrate patient-to-patient transmission of HCV during colonoscopy diagnosis in a Spanish hospital [86].

Secondly, viral phylogenetics and epidemiology allow us to address issues such as where and when did new viruses crossed the species boundary, identify reservoir species or even make predictions about viral emergence. Several general results have been reached in this field. For instance, viruses that cause acute infections frequently jump between host species and evolve rapidly, whereas viruses that cause persistent infections tend to infect a single host species and slowly coevolve with their host [84]. The former are usually RNA viruses whereas the latter tend to be DNA viruses, although there are exceptions to this rule. RNA viruses have caused several zoonoses in the past century, including among others, transmission of HIV-1 from chimpanzees to humans, swine/avian influenza pandemics, or the 2003 SARS outbreak (which involved bats as the putative reservoir hosts and palm civets as the source of transmission to humans) $[2,9]$. Evolutionary analysis combined with ecological, genetic and virological 
studies may allow us to build efficient surveillance programs in order to prevent pandemics in the future.

\section{Future perspectives}

Viral mutation rates have been measured using widely different strategies. They sometimes contain several sources of bias and error and are expressed using different, non-convertible, units. Moreover, in vitro and ex vivo viral mutation rate studies have frequently yielded disparate results. Our knowledge of in vivo rates is still very limited, but it would be not surprising that they differed from those obtained ex vivo and in vitro. Therefore, a more comprehensive empirical knowledge of viral mutation is necessary.

Evolutionary theory establishes a direct link between mutation and molecular evolutionary rates [87]. Broadly speaking, data obtained with viruses confirm this link, because RNA viruses mutate and evolve faster than DNA viruses. However, a finergrain correlation between these two parameters has not been demonstrated yet, possibly due to large estimation errors. More surprisingly, no systematic comparison has been undertaken yet between viral mutation and evolution rates.

Another important challenge is to clarify the forces driving the evolution of mutation rates. Particularly, the evolutionary basis for Drake's rule remains uncertain, and the mechanisms that allow ssDNA viruses to mutate significantly faster than their hosts or than dsDNA viruses remain to be elucidated in most cases. It is also unknown whether the inverse relationship between mutation rates and genome sizes can be extended to RNA viruses, and whether other factors such as genome polarity play a role in determining viral mutation and evolution.

The demonstration that the mutation rate of a virus determines its fitness and virulence is an important advance in the field. However, until very recently, most of this body of work built on a single RdRp variant and was restricted to members of the 
Picornaviridae family. Generalizing these results will strongly improve our understanding of viral pathogenesis and provide new basis for vaccine design.

Manipulating a virus' intrinsic mutation rate could be of potential benefit not only to live virus vaccine development, but to the production of inactivated virus vaccines as well. A high fidelity variant that replicates at wildtype levels will expectedly express larger quantities of correctly folded viral proteins, which could potentially increase production yields as well as elicit more specific immune responses. Alternatively, a lower fidelity variant with increased mutation rates may generate a more diverse population of antigens that might increase the likelihood of cross-protection against related strains presenting moderately different antigenic sites.

Lethal mutagenesis of viruses has a solid theoretical basis and has been extensively demonstrated in the laboratory. Although it has proved effective against many RNA viruses in cell cultures and occasionally in animal models, its usefulness in the clinical setting remains to be shown. In addition, resistance to this type of treatment and its potential perils should be carefully considered, given that alterations of the mutation rate may have important effects in viral adaptability, fitness, or virulence. More broadly speaking, new drugs should be evaluated not only for efficacy but also for the probability or resistance and its consequences.

Finally, a better understanding of how mutation rates are set and how they could be manipulated may open the way to using fidelity variants of RNA viruses to experimentally accelerate or decelerate evolution as a means of forecasting future strains emerging in response to developing immune responses within an individual and at the human population level. 


\section{Executive summary}

\section{Mechanisms of viral mutation}

- The absence of 3' exonuclease activity in most RdRps provides the clearest single mechanistic basis for the high mutation rates of RNA viruses, compared with DNA viruses.

- However, the mutation rate of a virus depends on processes other than the fidelity of its polymerase. Additional sources of mutations include spontaneous damage of the genetic material, proteins and co-factors that modulate polymerase fidelity, repair, or host-mediated editing.

\section{Measuring viral mutation rates}

- Viral mutation rates determined in vitro and ex vivo have frequently produced discrepant results. In vivo studies are far less abundant, yet necessary.

- Common problems in mutation rate estimation are the bias introduced by selection, sequencing errors, analysis of a few potentially non-representative genome sites, and the use of different definitions and measurement units.

\section{Variation in mutation rates across viruses}

- $\quad$ RNA viruses mutate faster than DNA viruses, ssDNA viurses mutate faster than dsDNA viruses, and mutation rates are inversely correlated with genome sizes across DNA viruses and microorganisms.

- Genome size and polarity play a less obvious role in determining mutation rates among RNA viruses.

\section{Viral mutation and virulence}

- A single amino acid replacement can simultaneously reduce the mutation rate and the virulence of a poliovirus in mice. A likely explanation for this result is that 
high fidelity variants have reduced adaptability. Recently, a similar finding has been obtained with a togavirus.

\section{Viral mutation and vaccines}

- RNA virus rapid mutation and evolution poses an important challenge to vaccine development, as illustrated by the cases of influenza virus, HIV-1 or HCV, although there also RNA viruses for which effective vaccines exist.

- Another downside of RNA virus high mutation rates is that live attenuated vaccines have the potential to revert to virulence. Attenuated high fidelity variants would have the advantage of reverting less often than currently used vaccines.

\section{Viral mutation and antiviral treatments}

- Although highly efficient in the short-term, most antiviral drugs fail in the longterm due the emergence of drug-resistant viruses.

- A potentially useful antiviral strategy consists of artificially increasing the genetic load of a virus using specific mutagens. This strategy, called lethal mutagenesis, has proved effective against many RNA viruses in controlled laboratory conditions, but its therapeutic value remains to be shown. 


\section{References}

1. Duffy S, Holmes EC. Phylogenetic evidence for rapid rates of molecular evolution in the single-stranded DNA begomovirus tomato yellow leaf curl virus. J. Virol. 82, 957-965 (2008).

2. Holmes EC. The evolution and emergence of RNA viruses. Oxford University Press (2009).

3. Sanjuán R, Nebot MR, Chirico N, Mansky LM, Belshaw R. Viral mutation rates. J. Virol. 84, 9733-9748 (2010).

4. Perelson AS. Modelling viral and immune system dynamics. Nat. Rev. Immunol. $2,28-36(2002)$.

5. Davenport MP, Loh L, Petravic J, Kent SJ. Rates of HIV immune escape and reversion: implications for vaccination. Trends Microbiol. 16, 561-566 (2008).

6. Vignuzzi M, Wendt E, Andino R. Engineering attenuated virus vaccines by controlling replication fidelity. Nat. Med. 14, 154-161 (2008).

7. Pfeiffer JK, Kirkegaard K. Increased fidelity reduces poliovirus fitness and virulence under selective pressure in mice. PLoS Pathog. 1, e11 (2005).

8. Vignuzzi M, Stone JK, Arnold JJ, Cameron CE, Andino R. Quasispecies diversity determines pathogenesis through cooperative interactions in a viral population. Nature 439, 344-348 (2006).

9. Pepin KM, Lass S, Pulliam JR, Read AF, Lloyd-Smith JO. Identifying genetic markers of adaptation for surveillance of viral host jumps. Nat. Rev. Microbiol. $8,802-813(2010)$.

10. Menéndez-Arias L. Mutation rates and intrinsic fidelity of retroviral reverse transcriptases. Viruses 1, 1137-1165 (2009). 
11. Arias A, Arnold JJ, Sierra M, et al. Determinants of RNA-dependent RNA polymerase (in)fidelity revealed by kinetic analysis of the polymerase encoded by a foot-and-mouth disease virus mutant with reduced sensitivity to ribavirin. $J$. Virol. 82, 12346-12355 (2008).

12. Arnold JJ, Vignuzzi M, Stone JK, Andino R, Cameron CE. Remote site control of an active site fidelity checkpoint in a viral RNA-dependent RNA polymerase. J. Biol. Chem. 280, 25706-25716 (2005).

13. Steinhauer DA, Domingo E, Holland JJ. Lack of evidence for proofreading mechanisms associated with an RNA virus polymerase. Gene 122, 281-288 (1992).

14. Minskaia E, Hertzig T, Gorbalenya AE, et al. Discovery of an RNA virus 3'->5' exoribonuclease that is critically involved in coronavirus RNA synthesis. Proc. Natl. Acad. Sci. USA 103, 5108-5113 (2006).

15. Denison MR, Graham RL, Donaldson EF, Eckerle LD, Baric RS. Coronaviruses: an RNA proofreading machine regulates replication fidelity and diversity. $R N A$. Biol. 8, 270-279 (2011).

16. Pfeiffer JK, Kirkegaard K. A single mutation in poliovirus RNA-dependent RNA polymerase confers resistance to mutagenic nucleotide analogs via increased fidelity. Proc. Natl. Acad. Sci. USA 100, 7289-7294 (2003).

17. Kunkel TA. The mutational specificity of DNA polymerase-beta during in vitro DNA synthesis. Production of frameshift, base substitution, and deletion mutations. J. Biol. Chem. 260, 5787-5796 (1985).

18. Lerner DL, Wagaman PC, Phillips TR, et al. Increased mutation frequency of feline immunodeficiency virus lacking functional deoxyuridine-triphosphatase. Proc. Natl. Acad. Sci. USA 92, 7480-7484 (1995). 
19. Mansky LM, Preveral S, Selig L, Benarous R, Benichou S. The interaction of vpr with uracil DNA glycosylase modulates the human immunodeficiency virus type 1 In vivo mutation rate. J. Virol. 74, $7039-7047$ (2000).

20. Harris RS, Bishop KN, Sheehy AM, et al. DNA deamination mediates innate immunity to retroviral infection. Cell 113, 803-809 (2003).

21. Lecossier D, Bouchonnet F, Clavel F, Hance AJ. Hypermutation of HIV-1 DNA in the absence of the Vif protein. Science 300, 1112 (2003).

22. Mangeat B, Turelli P, Caron G, et al. Broad antiretroviral defence by human APOBEC3G through lethal editing of nascent reverse transcripts. Nature 424, 99-103 (2003).

23. O'Hara PJ, Nichol ST, Horodyski FM, Holland JJ. Vesicular stomatitis virus defective interfering particles can contain extensive genomic sequence rearrangements and base substitutions. Cell 36, 915-924 (1984).

24. Cattaneo R, Schmid A, Eschle D, et al. Biased hypermutation and other genetic changes in defective measles viruses in human brain infections. Cell 55, 255-265 (1988).

25. Hajjar AM, Linial ML. Modification of retroviral RNA by double-stranded RNA adenosine deaminase. J. Virol. 69, 5878-5882 (1995).

26. Kim T, Mudry RA, Jr., Rexrode CA, Pathak VK. Retroviral mutation rates and A-to-G hypermutations during different stages of retroviral replication. J. Virol. 70, 7594-7602 (1996).

27. Sharmeen L, Bass B, Sonenberg N, Weintraub H, Groudine M. Tat-dependent adenosine-to-inosine modification of wild-type transactivation response RNA. Proc Natl. Acad. Sci. USA 88, 8096-8100 (1991). 
28. Domingo-Calap P, Sentandreu V, Bracho MA, et al. Unequal distribution of RTPCR artifacts along the E1-E2 region of Hepatitis C virus. J. Virol. Methods 161, 136-140 (2009).

29. Luria SE, Delbrück M. Mutations of bacteria from virus sensitivity to virus resistance. Genetics 28, 491-511 (1943).

30. Zheng Q. New algorithms for Luria-Delbruck fluctuation analysis. Math. Biosci. 196, 198-214 (2005).

31. Malpica JM, Fraile A, Moreno I, et al. The rate and character of spontaneous mutation in an RNA virus. Genetics 162, 1505-1511 (2002).

32. Tromas N, Elena SF. The rate and spectrum of spontaneous mutations in a plant RNA virus. Genetics 185, 989 (2010).

33. Gago S, Elena SF, Flores R, Sanjuán R. Extremely high mutation rate of a hammerhead viroid. Science 323, 1308 (2009).

34. Cuevas JM, González-Candelas F, Moya A, Sanjuán R. The effect of ribavirin on the mutation rate and spectrum of Hepatitis $\mathrm{C}$ virus in vivo. J. Virol. 83, 5760-5764 (2009).

35. Drake JW. A constant rate of spontaneous mutation in DNA-based microbes. Proc. Natl. Acad. Sci. USA 88, 7160-7164 (1991).

36. Shackelton LA, Parrish CR, Truyen U, Holmes EC. High rate of viral evolution associated with the emergence of carnivore parvovirus. Proc. Natl. Acad. Sci. USA 102, 379-384 (2005).

37. Shackelton LA, Holmes EC. Phylogenetic evidence for the rapid evolution of human B19 erythrovirus. J. Virol. 80, 3666-3669 (2006).

38. Duffy S, Shackelton LA, Holmes EC. Rates of evolutionary change in viruses: patterns and determinants. Nat. Rev. Genet. 9, 267-276 (2008). 
39. Chare ER, Gould EA, Holmes EC. Phylogenetic analysis reveals a low rate of homologous recombination in negative-sense RNA viruses. J. Gen. Virol. 84, 2691-2703 (2003).

40. Laengle-Rouault F, Maenhaut-Michel G, Radman M. GATC sequence and mismatch repair in Escherichia coli. EMBO J. 5, 2009-2013 (1986).

41. Cuevas JM, Pereira-Gómez M, Sanjuán R. Mutation rate of bacteriophage $\phi X 174$ modified through changes in GATC sequence context. Infect. Genet. Evol. 11, 1820-1822 (2011).

42. Chao L, Rang CU, Wong LE. Distribution of spontaneous mutants and inferences about the replication mode of the RNA bacteriophage $\phi 6 . J$. Virol. 76 , 3276-3281 (2002).

43. Gibson TC, Scheppe ML, Cox EC. Fitness of an Escherichia coli mutator gene. Science 169, 686-688 (1970).

44. Watson ME, Jr., Burns JL, Smith AL. Hypermutable Haemophilus influenzae with mutations in mutS are found in cystic fibrosis sputum. Microbiology 150, 2947-2958 (2004).

45. Richardson AR, Yu Z, Popovic T, Stojiljkovic I. Mutator clones of Neisseria meningitidis in epidemic serogroup A disease. Proc. Natl. Acad. Sci. USA 99, 6103-6107 (2002).

46. Mercier A, Bertolla F, Passelegue-Robe E, Simonet P. Natural transformationbased foreign DNA acquisition in a Ralstonia solanacearum mutS mutant. Res. Microbiol. 158, 537-544 (2007).

47. Schaaper RM. Mechanisms of mutagenesis in the Escherichia coli mutator mutD5: role of DNA mismatch repair. Proc. Natl. Acad. Sci. USA 85, 8126-8130 (1988). 
48. Levi LI, Gnadig NF, Beaucourt S, et al. Fidelity variants of RNA dependent RNA polymerases uncover an indirect, mutagenic activity of amiloride compounds. PLoS Pathog. 6, e1001163 (2010).

49. Coffey LL, Beeharry Y, Bordería AV, Blanc H, Vignuzzi M. Arbovirus high fidelity variant loses fitness in mosquitoes and mice. Proc. Natl. Acad. Sci. USA 108, 16038-16043 (2011).

50. Korneeva VS, Cameron CE. Structure-function relationships of the viral RNAdependent RNA polymerase: fidelity, replication speed, and initiation mechanism determined by a residue in the ribose-binding pocket. J. Biol. Chem. 282, 16135-16145 (2007).

51. Smith DJ, Lapedes AS, de Jong JC, et al. Mapping the antigenic and genetic evolution of influenza virus. Science 305, 371-376 (2004).

52. Korber BT, Letvin NL, Haynes BF. T-cell vaccine strategies for human immunodeficiency virus, the virus with a thousand faces. J. Virol. 83, 8300-8314 (2009).

53. Georgescu MM, Balanant J, MacAdam A, et al. Evolution of the Sabin type 1 poliovirus in humans: characterization of strains isolated from patients with vaccine-associated paralytic poliomyelitis. J. Virol. 71, 7758-7768 (1997).

54. Coen DM, Richman DD. Antiviral agents. In Fields Virology. Philadelphia: Lippincott-Raven. 447-486 (2012).

55. Menéndez-Arias L. Molecular basis of human immunodeficiency virus drug resistance: an update. Antiviral Res. 85, 210-231 (2010).

56. Moss RB, Davey RT, Steigbigel RT, Fang F. Targeting pandemic influenza: a primer on influenza antivirals and drug resistance. J. Antimicrob. Chemother. 65, 1086-1093 (2010). 
57. Domingo E Quasispecies: concept and implications for virology. Springer (2006).

58. Nijhuis M, Schuurman R, de JD, et al. Increased fitness of drug resistant HIV-1 protease as a result of acquisition of compensatory mutations during suboptimal therapy. AIDS 13, 2349-2359 (1999).

59. Quinones-Mateu ME, Moore-Dudley DM, Jegede O, Weber J, Arts J. Viral drug resistance and fitness. Adv. Pharmacol. 56, 257-296 (2008).

60. Ho DD. Time to hit HIV, early and hard. N. Engl. J. Med. 333, 450-451 (1995).

61. Orr HA. The rate of adaptation in asexuals. Genetics 155, 961-968 (2000).

62. Anderson JP, Daifuku R, Loeb LA. Viral error catastrophe by mutagenic nucleosides. Annu. Rev. Microbiol. 58, 183-205 (2004).

63. Bull JJ, Sanjuán R, Wilke CO. Theory of lethal mutagenesis for viruses. J. Virol. 81, 2930-2939 (2007).

64. Eigen M, McCaskill J, Schuster P. Molecular quasi-species. J. Phys. Chem. 92, 6881-6891 (1988).

65. Holland JJ, Domingo E, de la Torre JC, Steinhauer DA. Mutation frequencies at defined single codon sites in vesicular stomatitis virus and poliovirus can be increased only slightly by chemical mutagenesis. J. Virol. 64, 3960-3962 (1990).

66. Crotty S, Cameron CE, Andino R. RNA virus error catastrophe: direct molecular test by using ribavirin. Proc. Natl. Acad. Sci. USA 98, 6895-6900 (2001).

67. Graci JD, Harki DA, Korneeva VS, et al. Lethal mutagenesis of poliovirus mediated by a mutagenic pyrimidine analogue. J. Virol. 81, 11256-11266 (2007). 
68. Sierra S, Dávila M, Lowenstein PR, Domingo E. Response of foot-and-mouth disease virus to increased mutagenesis: influence of viral load and fitness in loss of infectivity. J. Virol. 74, 8316-8323 (2000).

69. Chung DH, Sun Y, Parker WB, et al. Ribavirin reveals a lethal threshold of allowable mutation frequency for Hantaan virus. J. Virol. 81, 11722-11729 (2007).

70. Grande-Pérez A, Lázaro E, Lowenstein P, Domingo E, Manrubia SC. Suppression of viral infectivity through lethal defection. Proc. Natl. Acad. Sci. USA 102, 4448-4452 (2005).

71. Dapp MJ, Clouser CL, Patterson S, Mansky LM. 5-Azacytidine can induce lethal mutagenesis in human immunodeficiency virus type 1. J. Virol. 83, 1195011958 (2009).

72. Loeb LA, Essigmann JM, Kazazi F, et al. Lethal mutagenesis of HIV with mutagenic nucleoside analogs. Proc. Natl. Acad. Sci. USA 96, 1492-1497 (1999).

73. Mullins JI, Heath L, Hughes JP, et al. Mutation of HIV-1 genomes in a clinical population treated with the mutagenic nucleoside KP1461. PLoS ONE 6, e15135 (2011).

74. Grande-Pérez A, Sierra S, Castro MG, Domingo E, Lowenstein PR. Molecular indetermination in the transition to error catastrophe: systematic elimination of lymphocytic choriomeningitis virus through mutagenesis does not correlate linearly with large increases in mutant spectrum complexity. Proc. Natl. Acad. Sci. USA 99, 12938-12943 (2002). 
75. González-López C, Gómez-Mariano G, Escarmís C, Domingo E. Invariant aphthovirus consensus nucleotide sequence in the transition to error catastrophe. Infect. Genet. Evol. 5, 366-374 (2005).

76. González-López C, Arias A, Pariente N, Gómez-Mariano G, Domingo E. Preextinction viral RNA can interfere with infectivity. J. Virol. 78, 3319-3324 (2004).

77. Manrubia SC, Domingo E, Lázaro E. Pathways to extinction: beyond the error threshold. Philos. Trans. R. Soc Lond B Biol. Sci. 365, 1943-1952 (2010).

78. Sierra M, Airaksinen A, González-López C, et al. Foot-and-mouth disease virus mutant with decreased sensitivity to ribavirin: implications for error catastrophe. J. Virol. 81, 2012-2024 (2007).

79. Agudo R, Ferrer-Orta C, Arias A, et al. A multi-step process of viral adaptation to a mutagenic nucleoside analogue by modulation of transition types leads to extinction-escape. PLoS Pathog. 6, e1001072 (2010).

80. Arribas M, Cabanillas L, Lázaro E. Identification of mutations conferring 5azacytidine resistance in bacteriophage Q $\beta$. Virology. 417, 343-352 (2011).

81. Sanjuán R, Cuevas JM, Furió V, Holmes EC, Moya A. Selection for robustness in mutagenized RNA viruses. PLoS Genet. 3, e93 (2007).

82. Perales C, Agudo R, Tejero H, Manrubia SC, Domingo E. Potential benefits of sequential inhibitor-mutagen treatments of RNA virus infections. PLoS Pathog. 5, e1000658 (2009).

83. Yeh SH, Wang HY, Tsai CY, et al. Characterization of severe acute respiratory syndrome coronavirus genomes in Taiwan: molecular epidemiology and genome evolution. Proc. Natl. Acad. Sci. USA 101, 2542-2547 (2004). 
84. Holmes EC. Evolutionary history and phylogeography of human viruses. Annu. Rev. Microbiol. 62, 307-328 (2008).

85. de OT, Pybus OG, Rambaut A, et al. Molecular epidemiology: HIV-1 and HCV sequences from Libyan outbreak. Nature 444, 836-837 (2006).

86. González-Candelas F, Guiral S, Carbo R, et al. Patient-to-patient transmission of hepatitis $\mathrm{C}$ virus (HCV) during colonoscopy diagnosis. Virol. J. 7, 217 (2010).

87. Kimura $\mathrm{M}$ The neutral theory of molecular evolution. Cambridge, Cambridge University Press (1983).

\section{Acknowledgments}

This work was financially supported by ERC Starting Grant 281191 to RS, grant BFU2011-25271 from the Spanish MICINN to RS and EL, grant FIS2011-27569 to EL, ERC Starting Grant 242719 to MV, and ANR 09-JCJC-0118 grant to MV. 


\section{Boxes and Figure legends}

\section{Box 1. Measuring viral mutation rates using the Luria-Delbrück fluctuation test}

Carrying out a Luria Delbrück fluctuation test requires a virus that can be plated and a selective agent in which only certain mutants can form visible plaques (a monoclonal antibody, an antiviral, a non-permissive cell type, etc.). A large number of replicate cultures are seeded with a small initial number of individuals $N_{i}$ each and after viral replication has occurred, $N_{f}$ viruses are plated in the presence of the selective agent. The statistical distribution of the number of resistant mutants per culture depends on the mutation rate, but also on the fitness effect of the mutations in the permissive medium and on the replication mechanism of the virus. A simple and convenient method for inferring the mutation rate that is largely independent of selection and replication mechanism is the so-called null-class method, which consists of counting the fraction of cultures with zero resistant mutants $\left(P_{0}\right)$. Since mutation is a rare event, the number of mutations per culture is expected to follow a Poisson distribution with parameter $\lambda=m\left(N_{f}-N_{i}\right)$, where $m$ is the mutation rate to from sensitivity to resistance. A property of this distribution is that $P_{0}=\exp \left(-m\left(N_{f}-N_{i}\right)\right)$. Once $m$ has been determined, mutants have to be sequenced to obtain the number of possible different mutations leading to the selected phenotype (mutation target size, $T$ ). The mutation rate can be expressed as substitutions per nucleotide per round of copying $(\mu)$ using the following equation: $\mu=3 m / T$ [3].

Figure 1. Schematic representation of a Luria-Delbrück fluctuation test (to be merged with Box 1). 
Figure 2. Viral mutation rate estimates obtained ex vivo and in vivo. Data are represented versus genome size and major virus groups are indicated. Values for viroids and bacteria, the two adjacent levels of biological complexity, are also plotted. Mutation rate is expressed as the number of substitutions per nucleotide per cell infection. Adapted from [3].

Figure 3. Effect of mutagenesis on a viral population. In RNA viruses, replication of a single genome rapidly gives rise to a genetically heterogeneous population often termed quasispecies. Cloning is often required to observe this variability, because at each given genome position the most frequent nucleotide or consensus is often the same as in the founder sequence. Chemically-induced mutagenesis increases the genetic variability of the population but may not alter the consensus sequence. Both the mutation frequency and the number of variants per site increase with time. Since most mutations are deleterious, this reduces the average fitness of the viral population and an extinction threshold may eventually be reached. 
Fig.1

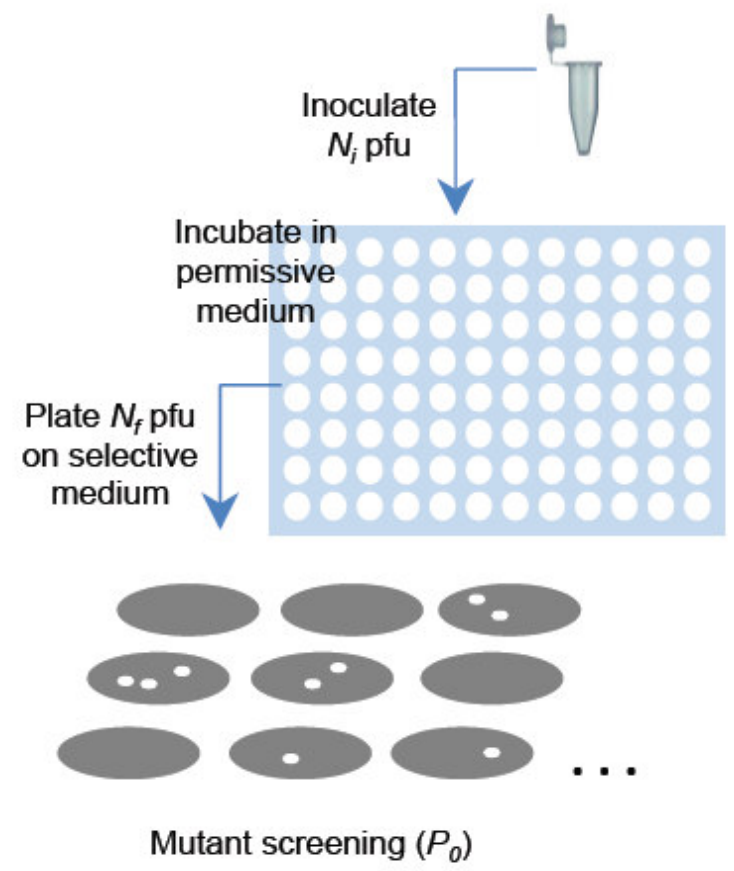


Fig. 2

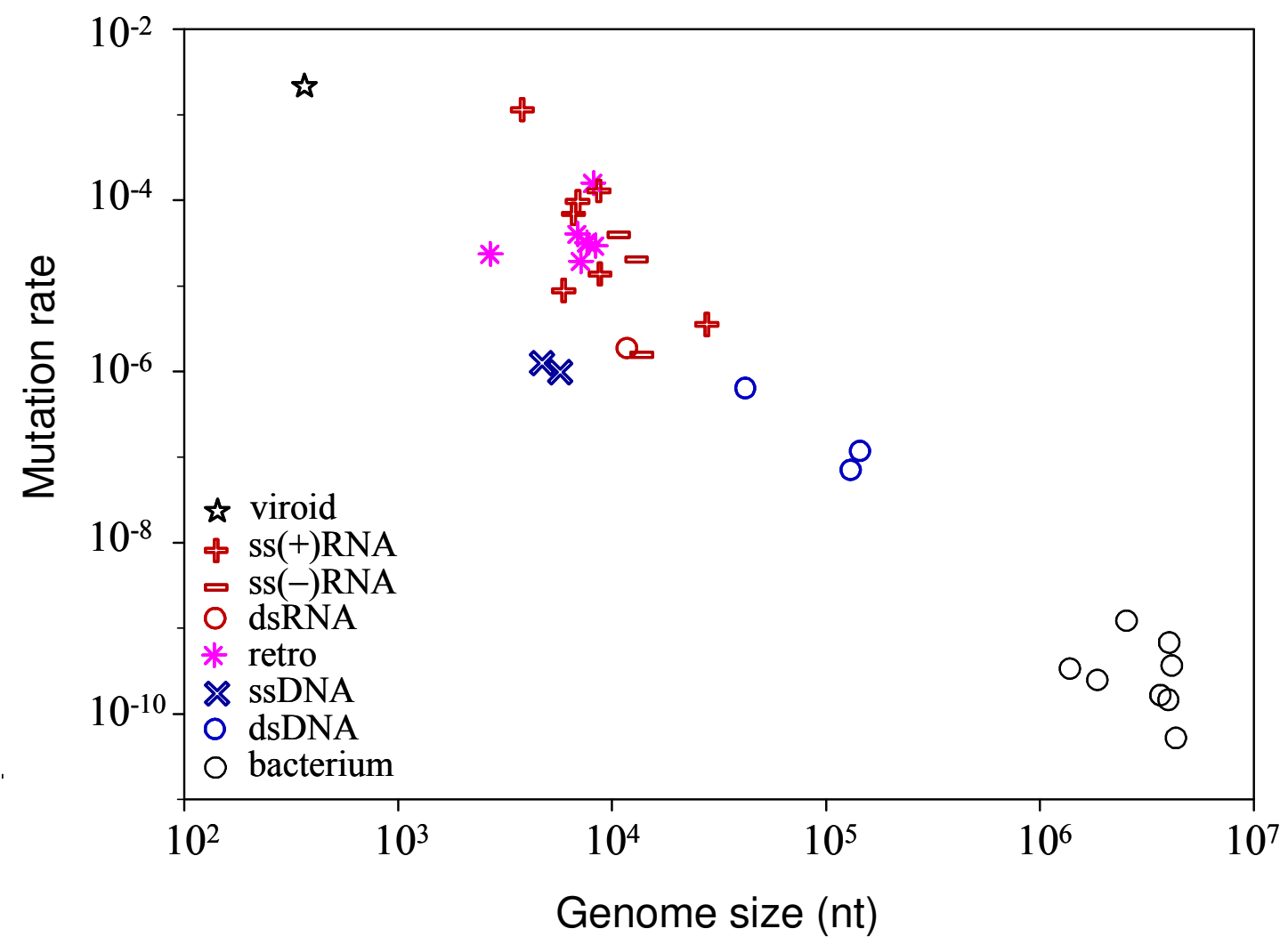


Fig. 3
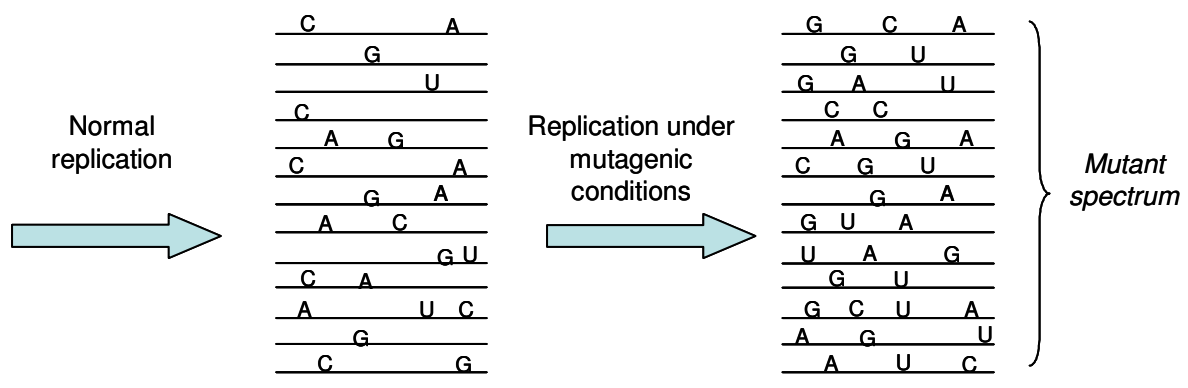

Consensus sequence



Generations in the presence of mutagen 